\title{
c-Met-Inhibitor bei NSCLC geprüft
}

Beim nichtkleinzelligen Lungenkarzinom (NSCLC) vom Nichtplattenepitheltyp konnte der experimentelle c-Met-Inhibitor Tivantinib unter bestimmten Bedingungen im Rahmen einer Phase-IIStudie das progressionsfreie Überleben verlängern. Nun ging die Prüfung in die Phase III. In der Studie wurde das Gesamtüberleben asiatischer NSCLC-Patienten mit einem Nichtplattenepithelkarzinom und Wildtyp-EGFR unter der Therapie mit Erlotinib plus Tivantinib oder Erlotinib plus Placebo untersucht.

Ursprünglich sollten 460 NSCLC-Patienten aufgenommen werden. Primärer Endpunkt war das Gesamtüberleben. Nach der Randomisierung von 307 Patienten wurde jedoch die weitere Aufnahme in die Studie gestoppt: Das Safety
Review Committee empfahl dies aufgrund des erheblichen Ungleichgewichts in der Inzidenz der interstitiellen Lungenkrankheit (ILD) zwischen den Gruppen. Im Tivantinib-Arm entwickelten 14 Patienten eine ILD, es kam zu drei Todesfällen. Im Placebo-Arm gab es sechs ILDPatienten und keinen Todesfall.

Bei den randomisierten Patienten lag das mittlere Gesamtüberleben bei 12,7 Monaten in der Tivantinib-Gruppe und bei 11,1 Monaten in der Placebogruppe (Hazard Ratio [HR] 0,891; $\mathrm{p}=0,427$ ). Das mittlere progressionsfreie Überleben lag bei 2,9 bzw. 2,0 Monaten (HR 0,719; $\mathrm{p}=0,019)$.

Die häufigsten Nebenwirkungen vom Schweregrad $\geq 3$ in der Tivantinib-Gruppe waren Neutropenie (24,3\%), Leukope-

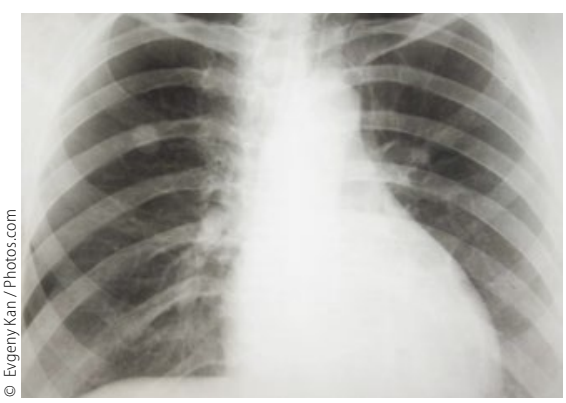

Bei der Therapie des NSCLC versucht man, in verschiedene Signalwege einzugreifen.

nie $(18,4 \%)$, febrile Neutropenie $(13,8 \%)$ und Anämie (13,2\%).

Christina Berndt

Yoshioka $\mathrm{H}$ et al. A randomized, double-blind, placebo-controlled, phase III trial of erlotinib with or without a c-Met inhibitor tivantinib (ARQ 197) in Asian patients with previously treated stage IIIB/IV nonsquamous nonsmall-cell lung cancer harboring wild-type epidermal growth factor receptor (ATTENTION study). Ann Oncol. 2015;26(10):2066-72.

\section{Endometriumkarzinom}

\section{Insulinresistenz steigert Krebsrisiko}

Die Wahrscheinlichkeit, ein Endometriumkarzinom zu entwickeln, ist bei Frauen mit einer Insulinresistenz deutlich erhöht. Das bestätigen die Ergebnisse einer US-amerikanischen Metaanalyse. Für diese konnten die Forscher die Daten von 25 Studien mit hoher Qualität einbeziehen. Insgesamt standen die Befunde von mehr als 33.000 Frauen zur Verfügung.

In 13 Studien mit mehr als 4000 Teilnehmerinnen waren die Nüchterninsulinspiegel bei Frauen mit einem Endometriumkarzinom signifikant höher als bei Frauen ohne diesen Krebs. Die mittlere Differenz betrug 33,94 pmol/1 ( $\mathrm{p}=$ 0,0004). Ebenfalls signifikant höher waren die C-Peptidspiegel (mittlere Differenz $0,14 \mathrm{nmol} / \mathrm{l} ; \mathrm{p}<0,00001$ ), basierend auf den Ergebnissen von fünf Studien. Schließlich ermittelten die Wissenschaftler auch den Parameter HOMA-IR (Homeostasis Model Assessment - Insulinresistenz) auf der Basis der Glukoseund Insulinwerte. Die Indexwerte als $\mathrm{Maß}$ für die Insulinresistenz waren bei Frauen mit Endometriumkarzinom signifikant höher als in der Vergleichsgruppe (mittlere Differenz 1,13; $\mathrm{p}=0,02$ ).
Bisher ist nicht klar, wie es durch chronisch erhöhte Insulinspiegel aufgrund einer Insulinresistenz zur Krebsentstehung kommt. Diskutiert wird u.a. der Einfluss (,insulin-like growth factor“)-I. Der Faktor ist vermehrt verfügbar, weil Insulin die Menge der IGF-Bindeproteine senkt. Er bindet an seinen Rezeptor, der häufiger auf Krebszellen vorkommt, und fördes Hormons auf die Wirkung des IGF dert dadurch z.B. die Zellproliferation und unterdrückt die Apoptose. Dies stimuliert schließlich die Karzinogenese. Insulin selbst werden ebenfalls mitogene Eigenschaften zugeschrieben. Krebs gilt Adipositas. Vor allem das Bauchfett fördert einen proentzündlichen Zustand im Gewebe, der wiederum auch die Entstehung einer Insulinresistenz begünstigt.

Peter Leiner

Hernandez AV et al. Insulin resistance and endometrial cancer risk: A systematic review and meta-analysis. Eur J Cancer. 2015;51(18):2747-58.
Als Bindeglied zwischen Diabetes und

\section{kurz notiert}

\section{Ovarialkarzinom: Muskulatur als Prognosemarker}

Bei Patientinnen mit epithelialem Ovarialkarzinom, die aufgrund des Tumors stark an Gewicht verlieren, ist die Kachexie ein wichtiger prognostischer Marker. Das haben Wiener Forscher nachgewiesen. Eine Tumorkachexie ist charakterisiert durch metabolische und inflammatorische Veränderungen, die sich in der Körperzusammensetzung widerspiegeln. Daher hatten die Wissenschaftler in ihrer Studie mithilfe der Computertomografie die Körperzusammensetzung von 140 Patientinnen mit epithelialem Ovarialkarzinom vor Therapiebeginn bestimmt, und zudem etablierte Marker für den Ernährungsund Entzündungsstatus sowie klinisch-pathologische Parameter. Dabei konnten sie zeigen, dass die Qualität der Skelettmuskulatur (LMA) sehr aussagekräftig für die Prognose der Patientinnen ist. Eine schwache Muskulatur war ein unabhängiger prognostischer Marker für das Überleben, mit einer Hazard Ratio von 2,25 ( $p=0,028$ ) Darüber hinaus war eine LMA signifikant mit dem Vorhandensein eines Resttumors nach der zytoreduktiven Operation $(p=0,046)$ und mit einem ungünstigen Performancestatus ( $p=0,015)$ assoziiert [Aust S et al. Plos One. 2015;10(10):e140403]. Judith Neumaier 